\title{
Cloning, chromosomal assignment and tissue distribution of human GILZ, a glucocorticoid hormone-induced gene
}

\section{Dear Editor,}

We have previously identified a murine leucine zippercontaining transcription factor, GILZ (GILZ: Glucocorticoid Induced Leucine Zipper) able to modulate $T$ lymphocyte activation and apoptosis. ${ }^{1,2}$ In the present study, we describe the isolation and cloning of a human homologue of murine GILZ (mGILZ). In particular, a $\lambda$ gt11 human cDNA library from $T$ lymphocytes was screened using the full length mGILZ cDNA, resulting in the identification of human GILZ (hGILZ). The human cDNA was $1946 \mathrm{bp}$ in total, consisting of a single open reading frame, beginning at nucleotide position 241 and extending to a termination codon at position 643 (Figure 1A). The first ATG of the hGILZ cDNA is surrounded by a minimum Kozak sequence (GAACCATGA) in good agreement with the consensus sequence for initiation of translation in eukaryotes. ${ }^{3}$ Besides, a typical polyadenylation signal, appears at the expected distance from the 3 '-terminus. The similarity of hGILZ to the nucleotide sequence to mGILZ is $89 \%$ in the entire mRNA and $97 \%$ in the coding region. The predicted translation product is a leucine zipper of 135 amino acids, highly homologous to mGILZ, with a putative molecular mass of about $15 \mathrm{kd}$ (Figure 1B). In particular, the encoded protein contains a leucine zipper-like structure from leucine76 to leucine-97, highly conserved among the members of this protein family. Furthermore, a proline rich region (PAR) is present at the $3^{\prime}$-end region. ${ }^{4}$ Structure analysis shows that the amino acid sequence in the leucine zipper region is hydrophilic at its C-terminus and hydrophobic at its $\mathrm{N}$ terminus. Consensus casein kinase-II phosphorylation sites are located at threonine- 8 , at serine- 88 (the start of its hydrophilic section), and at serine 114 . Besides, a protein kinase- $C$ consensus phosphorylation site at serine- 35 and a glycosylation consensus site at asparagine-19 are revealed.

We also determined the chromosomal localisation of hGILZ by fluorescence in situ hybridisation (FISH). ${ }^{5,6}$ For that purpose, metaphase spreads were obtained from human PHA-stimulated peripheral blood lymphocytes. Chromosome preparations were hybridised in situ using the entire hGILZ cDNA as a probe. Since the DAPI banding was used to identify the specific chromosome, the assignment between signal from the probe and long arm of chromosome $X$ was obtained (Figure 1C). The detailed position was determined in the diagram based on the summary from 10 photographs (Figure $1 \mathrm{Ca}$ and $\mathrm{b}$ ). Figure 1Ca shows a typical hybridisation of the hGILZ CDNA at the Xq22.2 region, of the chromatids of both chromosomes (Figure 1Ca and $\mathrm{Cc}$ arrow). Interestingly enough, the Xq22 region contains a number of genes associated with various $X$-linked deficiences including the Bruton agammaglobulinemia, further suggesting a possible role of GILZ in the regulation of immune response. Future studies aimed to identify the chromosomal localisation of mGILZ could furnish indications on the possible role in $\mathrm{X}$-linked deficiencies.

To determine the expression pattern of hGILZ gene, we performed Northern blot experiments with human tissue total RNA. ${ }^{7}$ Results of a representative experiment in Figure 1D indicate that hGILZ transcripts can be detected ubiquitously among different organs with the exception of liver (lane 4) and pancreas (lane 6). As shown in the figure, after $24 \mathrm{~h}$ of autoradiography, hGILZ RNA is clearly detectable in the brain, lung, spleen and skeletal muscle, while expression in heart and kidney is weaker. Evaluation of equal loading was performed by visual inspection of ethidium bromide (data not shown). This finding strongly indicates a widespread distribution of hGILZ and further suggests its possible role as transcription regulator in different tissues. ${ }^{2}$

We also analysed, by Northern blot analysis, the steady state levels of hGILZ RNA in a panel of human cell lines representative of different haematopoietic cell lineages (Figure 1Ea). hGILZ RNA messenger was highly expressed in the T cell line HUT-78 and the myeloid cell line HL-60, while expression was low in the monocyte cell lines THP1 and U937, the B cell lines JD38 and NAMALWA and undetectable in the lymphoblastic $T$ cell lines MOLT-3 and MOLT-4. Again, equal RNA loading was assessed by ethidium bromide staining of the gel (data not shown).

To further investigate the pattern of GILZ expression, we performed parallel Western blot analysis, using a rabbit polyclonal anti-mGILZ Ab. ${ }^{1}$ Results show a complete concordance between mRNA and protein expression, suggesting that GILZ expression is regulated rather at the transcriptional than at the post-transcriptional level (Figure 1Eb).

These observations led us to investigate hGILZ distribution in normal hematopoietic cells (Figure 1F). For this scope we examined the expression of hGILZ mRNA in bone marrow (BM) cells, CD34 ${ }^{+}$haematopoietic stem cells, monocytes, granulocytes, $\mathrm{PBL}, \mathrm{B}$ cells, and $\mathrm{CD}^{+} \mathrm{T}$ cells from human healthy donors by RT-PCR. ${ }^{8-10}$ The results in Figure $1 \mathrm{~F}$ show that hGILZ mRNA was present in BM, $\mathrm{CD} 34^{+}$stem cells, mature $\mathrm{B}$ and $\mathrm{T}$ lymphocytes, monocytes and granulocytes. Moreover, hGILZ expression was increased in $\mathrm{CD}_{4} 4^{+}$stem cells as compared to total BM or PBL in toto (from donors treated with G-CSF) indicating an enrichment of GILZ-expressing cells in the staminal cell fraction (Figure 1Fa).

We have previously shown that mGILZ is up-regulated in mouse thymocytes and lymphocytes by the synthetic glucocorticoid hormone dexamethasone (DEX). ${ }^{1}$ We 


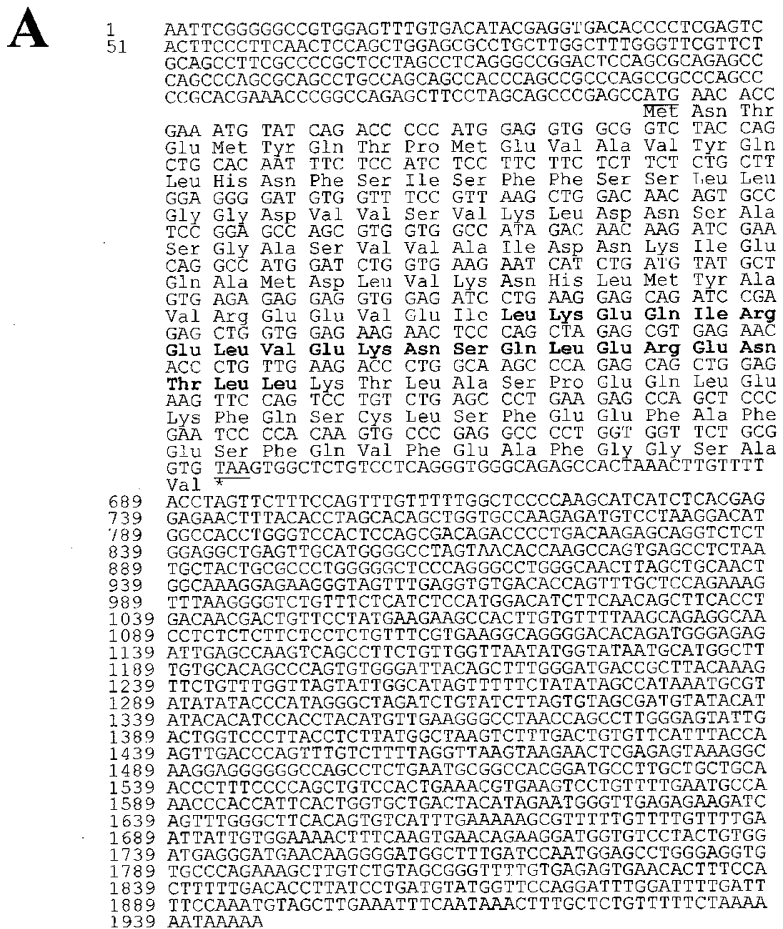

B

$$
\begin{aligned}
& \text { m-GILZ } 1 \text { MNTEMYQTPMEVAVYQLHNFSTSFFSSLLGGDVVSVKLDNSASGASVVAL } 50 \\
& \begin{aligned}
111111111111111111111.111111111111111111111111111: \\
\text { h-GILZ } 1 \text { MNTEMYQTPMEVAVYQLHNFSISFFSSLLGGDVVSKLDNSASGASVVAI } 50
\end{aligned} \\
& 51 \text { DNK IEQAMDLVKNHLMYAVREEVEVEKEQIRELLEKNSQLERENTLIKTL } 100 \\
& 111111111111111111111111: 11111111: 1111111111111111 \\
& 51 \text { DNKI IEQAMDLVKNHLMYAVREEVEIEKEQIRELVEKNSQLERENTLLKTL } 100 \\
& 101 \text { ASPEQLEKFQSRLSPEEPAPEAPETPETPEAPGGSAV* } 138 \\
& 11111111111|1| 1 \mid 1111 \text {. |:.11111111111 }
\end{aligned}
$$

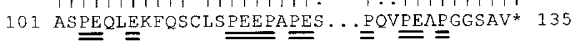

C

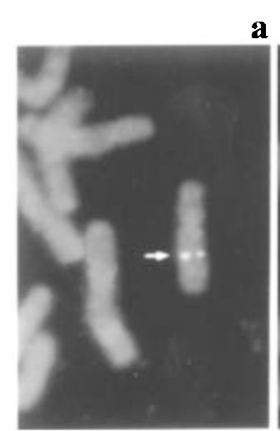

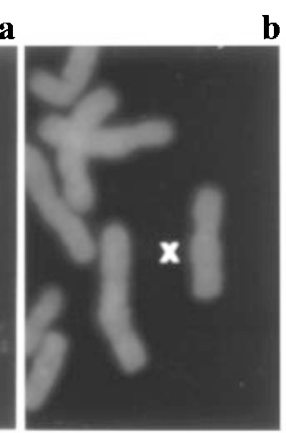

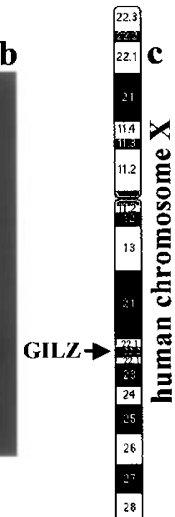

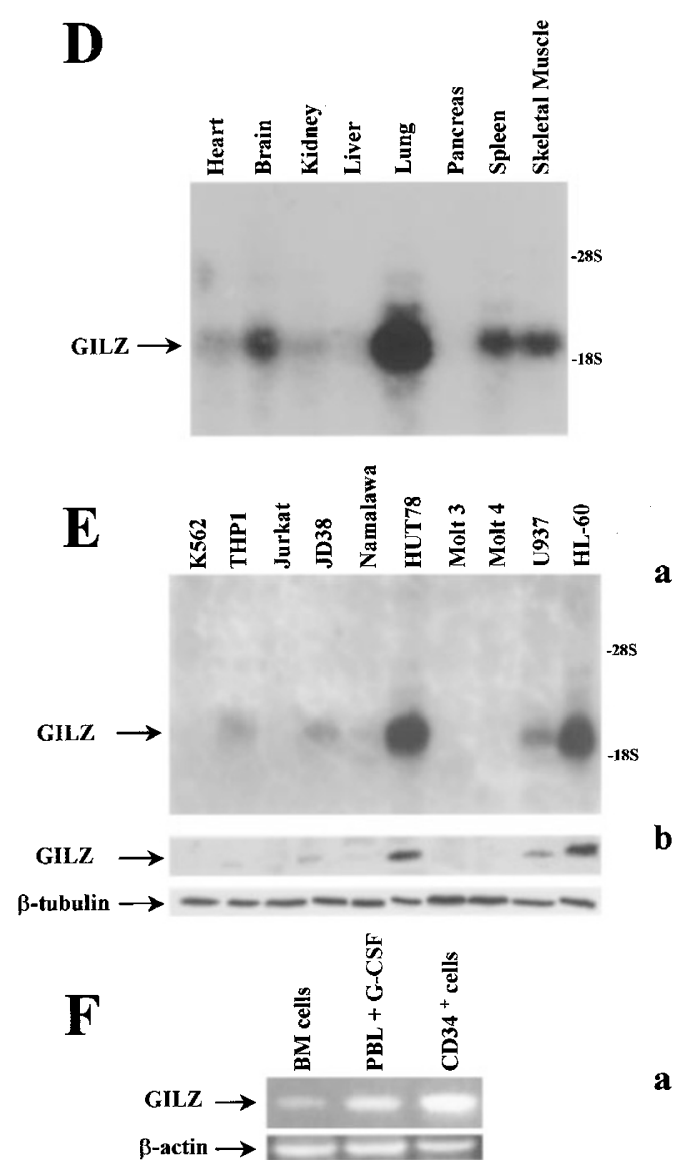

a

b

Figure 1 (A) Nucleotide and amino acid sequences of hGILZ. The leucine zipper region is evidenced in bold. (B) Comparison of the amino acid sequences of hGILZ and mGILZ. Open box, the leucine zipper domain; underlined, basic residues; double underlined, proline and acidic residues of the PAR region. (C) Chromosomal assignment to chromosome X. (a) the FISH signals on the chromosome. (b) The same mitotic figure stained with DAPI to identify chromosome X. (c) Diagram of FISH mapping result of hGILZ. (D) Northern blot analysis of hGILZ. $20 \mu \mathrm{g}$ of RNA fractionated on $1 \%$ denaturing agarose gel, transferred to a nitrocellulose filter and hybridised to ${ }^{32} \mathrm{P}$-labelled probe for hGILZ, washed and exposed for autoradiography for $24 \mathrm{~h}$. (E) Expression of $\mathrm{hGILZ} \mathrm{RNA} \mathrm{in} \mathrm{human} \mathrm{cell}$ lines. (a) Northern blot analysis. (b) Western blot analysis from total protein extracts performed with rabbit immune antiserum (top panel) or with anti- $\beta$-tubulin antiserum (bottom panel) as control. (b) hGILZ expression in normal lymphohaemopoietic subpopulations ( $>95 \%$ pure) as evaluated by semiquantitative RTPCR. (a) Analysis of GILZ expression (top panel) using RNA from human BM (lane 1), PBL stimulated with G-CSF (lane 2) and CD34 ${ }^{+}$cells from GM-CSFstimulated PBL (lane 3). b-actin control (bottom panel). (b) RT-PCR evaluation of hGILZ expression in normal lymphocytes. RNA from total PBL (lane 1), CD3 ${ }^{+}$ cells (lane 2), B cells (lane 3), monocytes (lane 4), granulocytes (lane 5) on the top panel, and $\beta$-actin on the bottom panel. (G) Effect of DEX on GILZ expression. RT-PCR from cells untreated or treated with DEX $\left(10^{-7} \mathrm{M}\right)$ for $3 \mathrm{~h}$ (top panel, $\beta$-actin bottom panel). Amplification of cDNA was carried out using hGILZ and $\beta$-actin specific primers: 5'-AATCCCCACAAGTGCCCGAGGCC-3' forward and 5'-ATTTACCAAGTTGACCCAGTTTGTC-3' reverse, for hGILZ; 5'-CTCGTCGTCGACAACGGCCTCC-3' forward and $5^{\prime}$-CTTCTCCTTAATGTCACGCACGAT- $3^{\prime}$ reverse, for $\beta$-actin. PCR conditions were as follows: $95^{\circ} \mathrm{C}$ for $50 \mathrm{~s}, 62^{\circ} \mathrm{C}$ for $50 \mathrm{~s}$ and $72 \mathrm{C}$ for $2 \mathrm{~min}$. PCR products were separated by electrophoresis on a $1.2 \%$ agarose gel and stained with ethidium bromide 
treated human $\mathrm{PBL}$, monocytes and $\mathrm{CD}^{+}$cells with $\mathrm{DEX}$, $10^{-7} \mathrm{M}$ for $3 \mathrm{~h}$. Results indicate that also hGILZ expression is increased by treatment with DEX (Figure $1 G)$, suggesting that, like mGILZ, hGILZ could be involved in the regulation of lymphohaemopoietic cell activation and death. $^{2}$

In conclusion we have identified hGILZ and have investigated its expression pattern. Our results indicate that this leucine zipper factor is also expressed in human lymphohaemopoietic cells and that its expression is modulated by treatment with DEX. Future studies will be devoted to analyse the role of hGILZ in regulating the development of bone marrow derived cells.

We are grateful to SeeDNA Biotech Inc. for performing the chromosomal localisation of hGILZ. This work was supported by Associazione Italiana Ricerca sul Cancro (AIRC), Milan and by CNR target project on Biotechnology, Rome, Italy. hGILZ gene bank accession number: AF228339.
L Cannarile ${ }^{1}$, O Zollo ${ }^{1}, F$ D'Adamio $^{1}$, E Ayroldi $^{1}$, C Marchetti $^{1}$, A Tabilio $^{1}$, Bruscoli $^{1}$ and C Riccardi ${ }^{*, 1}$

1 Department of Clinical and Experimental Medicine, Section of Pharmacology, Perugia University Medical School, 06100 Perugia, Italy

* Corresponding author: C Riccardi, Department of Clinical and Experimental Medicine, Section of Pharmacology, Perugia University Medical School, 06100 Perugia, Italy. E-mail: riccardi@unipg.it

1. D'Adamio $F$ et al (1997) Immunity 7: 803-812

2. Riccardi $C$ et al (1999) Cell Death Differ. 6: 1182-1189

3. Kozak O (1991) J. Biol. Chem. 266: 19867-19870

4. Drolet DW et al (1991) Genes Dev. 5: 1739-1753

5. Heng HHQ et al (1992) Proc. Natl. Acad. Sci USA 89: 9509-9513

6. Heng HHQ and Tsui L-C (1993) Chromosoma 102: 325-332

7. Nocentini $\mathrm{G}$ et al (1997) Proc. Natl. Acad. Sci. USA 94: 6216-6221

8. Carlo-Stella C et al (2000) Exp. Hematol. 28: 216-224

9. Irving SG et al (1989) Mol. Cell. Biol. 9: 1034-1039

10. Nocentini $G$ et al (2000) Cell Death Differ. 7: 408-410 